\title{
Analytical study on emittance growth caused by roughness of a metallic photocathode
}

\author{
Zhe Zhang and Chuanxiang Tang* \\ Department of Engineering Physics, Tsinghua University, Beijing 100084, China
}

(Received 6 September 2014; published 29 May 2015)

\begin{abstract}
The roughness of a photocathode could lead to an additional uncorrelated divergence of the emitted electrons and therefore to an increased thermal emittance. To calculate the emittance growth due to the cathode roughness, people usually choose a simple 2D sinusoidal surface model to avoid mathematical complexity. In this paper, we demonstrate an analytical method, which is inspired by the point spread function that has been widely used in radiation imaging field, to accurately evaluate the emittance growth due to the random roughness of a real-life cathode. Both analytical and numerical studies are performed. Our analytical formulas clearly reveal the relationship between the surface roughness and the emittance growth. Both analytical and numerical results surprisingly show that in the typical 3D random surface case, the influence of the surface roughness on the emittance growth is much smaller than the 2D sinusoidal case with typical roughness properties, however with roughness properties which are matched to the 3D case, the emittance growth conditions in these two cases are very similar. Even with applied electric field strength up to $120 \mathrm{MV} / \mathrm{m}$, the total emittance growth is still below $10 \%$. It implies that the large emittance growth $(50 \%-100 \%)$ observed on metallic cathodes in some experiments, which is generally believed to be the result of the electric field on the rough surface, might be due to some other reasons.
\end{abstract}

DOI: 10.1103/PhysRevSTAB.18.053401

PACS numbers: 85.60.Ha, 68.35.Ct, 29.27.-a, 79.60.-i

\section{INTRODUCTION}

A photocathode is an electrode that is able to produce high brightness electron beam by photoelectric effect. The capability of photocathodes to produce low emittance, high charge and controllable bunches is crucial to modern light sources. Photocathodes are widely integrated in large particle sources such as Linac Coherent Light Source (LCLS) [1], compact and high duty factor free electron laser [2,3], as well as many other light sources [4,5]. The most important characteristics that measure the quality of a photocathode are quantum efficiency (QE) and intrinsic emittance (or thermal emittance) of the emitted electron beam. The requirement for high brightness electron beam in modern light sources has pushed the development of photocathodes to achieve higher QE and lower thermal emittance. Dowell gives formulas [6] to predict the $\mathrm{QE}$ and thermal emittance of a metallic smooth surface photocathode by using a simplified three-step model [7]:

$$
\mathrm{QE}(\omega) \approx \frac{1-R(\omega)}{1+\frac{\lambda_{\mathrm{opt}}}{\lambda_{\mathrm{e} e \mathrm{e}}(\omega)}} \frac{\left(\hbar \omega-\phi_{\mathrm{eff}}\right)^{2}}{8 \phi_{\mathrm{eff}}\left(E_{F}+\phi_{\mathrm{eff}}\right)}
$$

\footnotetext{
*tang.xuh@tsinghua.edu.cn

Published by the American Physical Society under the terms of the Creative Commons Attribution 3.0 License. Further distribution of this work must maintain attribution to the author $(s)$ and the published article's title, journal citation, and DOI.
}

$$
\varepsilon_{n, x}=\sigma_{x} \sqrt{\frac{\hbar \omega-\phi_{\mathrm{eff}}}{3 m c^{2}}}
$$

Equation (1a) agrees well with the measurements taken on copper photocathodes, while Eq. (1b) has a significant deviation from the experimental results in many laboratories. Taking the nominal $\phi_{\mathrm{w}}=4.65 \mathrm{eV}$ as the work function of polycrystalline copper [8], the emittance measurement results from some labs are about 2 times larger than the formula prediction $[9,10]$. One possibility for thermal emittance data divergence is the variation of cathode surface condition [11]. Therefore the underestimation on thermal emittance of Eq. (1b) could be caused by the failure of smooth surface assumption. In other words, the roughness of the cathode has contributed to the growth of the thermal emittance.

Generally speaking, the emittance growth caused by the surface roughness could be divided into two types: the emittance growth due to the emission angle diffusion ("slope effect") $[12,13]$, and the emittance growth induced by the transverse electric field comes from the applied electric field on the rough surface ("field effect") [12-15]. To evaluate the total amount of emittance growth, people usually apply a 2D or 3D sinusoidal model to get approximate formulas for each type of emittance growth separately, then ignore slope effect related emittance growth since it is rather small compared to the field effect related emittance growth, and finally calculate the total emittance by using the following equation $[16,17]$ : 


$$
\varepsilon_{n, \text { total }}=\sqrt{\varepsilon_{n, \text { smooth }}^{2}+\varepsilon_{n, \text { field }}^{2}}
$$

with the assumption that the smooth surface emittance and the field effect related emittance are independent. While this simple method has been successfully used to qualitatively explain the intrinsic emittance growth due to the surface roughness, it lacks the power to predict the experimental results because the surface roughness model employed is too simplified, as well as the neglect of the cross term between the smooth surface emittance and the field effect related emittance.

The main purpose of this paper is to accurately calculate the total emittance growth caused by the roughness of a metallic photocathode. To achieve that, one has to obtain the accurate phase-space distribution of the electrons emitted from the surface of the photocathode irradiated by a laser beam. That is the reason why we developed the point spread function (PSF) method. The rest of this paper is organized as follows: Section II introduces the point spread function of a photocathode, and uses it as a tool to examine the validation of the assumption that the electron momentum distribution is independent of the emitted position. Section III presents the derivation of the formulas for emittance growth induced by the roughness of the photocathode. Section IV describes the principles of numerical simulation and discusses the simulation results.

It should be noted that this paper used the bulk emission model which is employed in Dowell's work [6,7], hence the formulas shown in the following sections are only valid in the bulk emission case if not mentioned. Nevertheless, the idea that using the point spread function to calculate the roughness emittance is model independent thus has potential to be applied to a wider range of situations.

\section{POINT SPREAD FUNCTION OF PHOTOCATHODE}

To predict the image on an uniform screen when irradiated by a laser beam, we just need to know the intensity distribution of the laser beam, and the image on the screen irradiated at a single point. The latter is called point spread function (PSF) of the screen, which describes how the screen respond to single point incidence. Assuming the PSF of the screen has the form of $f(x, y)$, then the image of the screen irradiated by a laser beam with intensity distribution $I(x, y)$ would be

$$
D(x, y)=I(x, y) * f(x, y)
$$

which means that the image on the screen is the convolution of the incident laser intensity distribution and the PSF of the screen.

A similar concept could be applied on calculations on the response of a photocathode to the incident laser, which reveals the emitted electron phase-space distribution. The

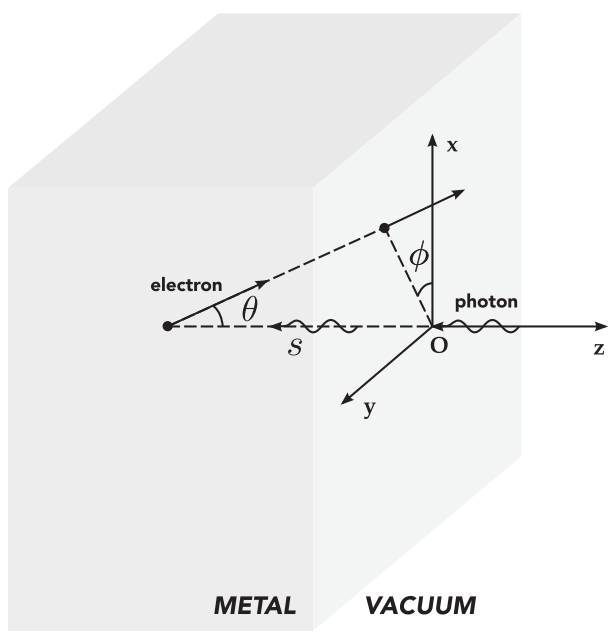

FIG. 1. The schematic plot for the bulk photoemission on a smooth metallic cathode. The definitions of coordinates and variables are marked in the plot.

only difference between the PSF of a photocathode and the PSF of a screen is that the screen PSF only focuses on the real-space distribution, while the photocathode PSF cares about the phase-space distribution.

\section{A. Electrons phase-space distribution caused by normal incidence at a single point}

To derive the photocathode PSF, we pick up the cathode model the same as what Dowell has used: a bulk emission, smooth surface metallic photocathode model, of which the coordinates configuration is shown in Fig. 1.

Following the same derivations of Dowell's formula, one could get the possibility of one normal incident photon injected into the bulk metal, after traveling a distance of $s$, absorbed by an electron of energy $E$, and went towards the surface with a direction angle $(\theta, \phi)$ without scattering and finally escaped from the surface, as Eq. (3) [6].

$$
\begin{aligned}
f(s, \theta, \phi, E, \omega)= & (1-R) \frac{1}{\lambda_{\mathrm{opt}}} \exp \left[-s\left(\frac{1}{\lambda_{\mathrm{opt}}}+\frac{1}{\bar{\lambda}_{\mathrm{e}-\mathrm{e}}}\right)\right] \\
& \cdot \frac{H\left(E_{\mathrm{F}}-E\right) H\left(E+\hbar \omega-E_{\mathrm{F}}\right)}{\hbar \omega} \frac{\sin \theta}{4 \pi} .
\end{aligned}
$$

The $H(x)$ function in Eq. (3) is a Heaviside function, to simplify the form, let

$$
\begin{aligned}
\frac{1}{\lambda} & =\frac{1}{\lambda_{\mathrm{opt}}}+\frac{1}{\bar{\lambda}_{\mathrm{e}-\mathrm{e}}}, \quad C=\frac{1-R}{\lambda_{\mathrm{opt}} \cdot 4 \pi \hbar \omega}, \\
p_{m} & =\sqrt{2 m\left(E_{F}+\phi_{\mathrm{eff}}\right)}, \quad p_{M}=\sqrt{2 m\left(E_{F}+\hbar \omega\right)},
\end{aligned}
$$

with consideration of the relations between $(s, \theta, \phi, E, \omega)$ and $\left(x, y, p_{x}, p_{y}, p_{z}\right)$ : 


$$
\begin{aligned}
x & =s \tan \theta \cos \phi, \quad y=s \tan \theta \sin \phi, \\
p_{x} & =\sqrt{2 m(E+\hbar \omega)} \sin \theta \cos \phi, \\
p_{y} & =\sqrt{2 m(E+\hbar \omega)} \sin \theta \sin \phi, \\
p_{z} & =\sqrt{2 m(E+\hbar \omega) \cos ^{2} \theta-2 m\left(E_{F}+\phi_{\text {eff }}\right)} .
\end{aligned}
$$

One could obtain the PSF of the photocathode as shown in Eq. (6) (details in Appendix A):

$$
\begin{aligned}
f\left(x, y, p_{x}, p_{y}, p_{z}\right)= & C \exp \left[-\frac{\sqrt{p_{z}^{2}+p_{m}^{2}}}{\sqrt{p_{x}^{2}+p_{y}^{2}}} \cdot \frac{\sqrt{x^{2}+y^{2}}}{\lambda}\right] \\
& \cdot \frac{p_{z}}{\sqrt{p_{x}^{2}+p_{y}^{2}+p_{z}^{2}+p_{m}^{2}}} \cdot \delta\left(x p_{y}-y p_{x}\right) \\
& \cdot H\left(p_{z}\right) H\left(x p_{x}\right) \\
& \times H\left(p_{M}^{2}-p_{m}^{2}-p_{x}^{2}-p_{y}^{2}-p_{z}^{2}\right) .
\end{aligned}
$$

By using PSF Eq. (6), we could easily calculate the phase-space distribution of the electron beam that was extracted by laser beam of any intensity distribution. For example, the phase-space distribution function $G(\boldsymbol{x}, \boldsymbol{p})$ for the electron bunch ejected by a laser with Gaussian transverse intensity distribution,

$$
I(x, y)=\frac{I_{0}}{2 \pi \sigma_{x} \sigma_{y}} \cdot \exp \left[-\left(\frac{x^{2}}{2 \sigma_{x}^{2}}+\frac{y^{2}}{2 \sigma_{y}^{2}}\right)\right],
$$

has the form shown in Eq. (7):

$$
\begin{aligned}
G & =\frac{\sqrt{\pi}}{2} \frac{1}{\sqrt{\frac{p_{x}^{2}}{2 \sigma_{x}^{2}}+\frac{p_{y}^{2}}{2 \sigma_{y}^{2}}}} \exp \left[-\frac{x^{2}}{2 \sigma_{x}^{2}}-\frac{y^{2}}{2 \sigma_{y}^{2}}+T^{2}\right] \cdot \operatorname{erfc}(T), \\
T & =\frac{-\frac{x p_{x}}{2 \sigma_{x}^{2}}-\frac{y p_{y}}{2 \sigma_{y}^{2}}+\frac{\sqrt{p_{z}^{2}+p_{m}^{2}}}{2 \lambda}}{\sqrt{\frac{p_{x}^{2}}{2 \sigma_{x}^{2}}+\frac{p_{y}^{2}}{2 \sigma_{y}^{2}}}} .
\end{aligned}
$$

\section{B. The accuracy of the Dowell formula for emittance of smooth photocathode}

The statistical definition of emittance is described in Eq. (8) [18]:

$$
\varepsilon_{D, x}^{2}=\left\langle x^{2}\right\rangle_{D}\left\langle p_{x}^{2}\right\rangle_{D}-\left\langle x p_{x}\right\rangle_{D}^{2} .
$$

The symbol $D$ in Eq. (8) stands for distribution of the electron phase space. In derivation of Dowell's formula for emittance, the $\left\langle x p_{x}\right\rangle$ term was ignored with the assumption that the electron momentum distribution is independent of the photon incident position. It is also assumed that the emitted electron real-space distribution was the same as the laser intensity distribution. With these assumptions the emittance was simplified to $\varepsilon_{x}=\sqrt{\left\langle x^{2}\right\rangle\left\langle p_{x}^{2}\right\rangle}=\sigma_{x} \sqrt{\left\langle p_{x}^{2}\right\rangle}$, so that $\varepsilon_{x, n}=\sigma_{x} \cdot \Delta_{x}, \Delta_{x}$ is the $x$ direction normalized divergence which is defined as $\sqrt{\left\langle p_{x}^{2}\right\rangle} / m c$. With the PSF we just derived, one could check the accuracy of the Dowell's emittance formula by calculating the statistical terms in Eq. (8).

Supposed that we have a variable $v$ in the form $v\left(x, y, p_{x}, p_{y}, p_{z}\right)$, to evaluate the expectation of $v$ under the probability density distribution $D=I * f$, there is a rule shown in Eq. (9) (derivation for a simplified version could be found in Appendix D 1):

$$
\langle v\rangle_{D}=\langle v\rangle_{I * f}=\left\langle v\left(x_{I}+x_{f}, y_{I}+y_{f}, p_{x}, p_{y}, p_{z}\right)\right\rangle_{I \cdot f} .
$$

The $x$ and $y$ with subscript in Eq. (9) means that they are variable only under the distribution in their subscript, say, $x_{I}$ is a constant when calculate $\left\langle x_{I}\right\rangle_{f}$ but is a variable when calculate $\left\langle x_{I}\right\rangle_{I}$. Also note that the symbol $\langle\cdots\rangle_{I . f}$ actually stands for nesting average $\left\langle\langle\cdots\rangle_{I}\right\rangle_{f}$ or $\left\langle\langle\cdots\rangle_{f}\right\rangle_{I}$, since swapping the subscripts of the inner and outer brackets will not change the statistic result, we simplify the nesting average symbol as $\langle\cdots\rangle_{I \cdot f}$.

After a certain amount of calculations, one could obtain

$$
\varepsilon_{D, x}^{2}=\left\langle x^{2}\right\rangle_{I}\left\langle p_{x}^{2}\right\rangle_{f}+\varepsilon_{f}^{2},
$$

where $\varepsilon_{f}^{2}$ is defined as $\left\langle x^{2}\right\rangle_{f}\left\langle p_{x}^{2}\right\rangle_{f}-\left\langle x p_{x}\right\rangle_{f}^{2}$ which stands for the single point emittance. The first term on the right side of Eq. (10) is equivalent of the emittance formula derived by Dowell, so the second term $\varepsilon_{f}$ would be the deviation of Dowell's formula. When $\hbar \omega$ is near $\phi_{\text {eff }}$ (which is the typical situation), $\varepsilon_{f}$ could be written as the approximate form below (details in Appendix D 2):

$$
\varepsilon_{f} \approx \frac{1}{3} \lambda\left(p_{M}-p_{m}\right)
$$

in which $p_{M}, p_{m}$ and $\lambda$ keep the same definitions as in Eq. (4). $\left\langle p_{x}^{2}\right\rangle_{f}$ could be written as (derivation also in Appendix D 2)

$$
\left\langle p_{x}^{2}\right\rangle_{f}=\frac{1}{6}\left(p_{M}^{2}-p_{m}^{2}\right) .
$$

One could obtain the approximate form of the emittance as shown in Eq. (11) (keep the first order quantity):

$$
\varepsilon_{D, x}^{2} \approx \varepsilon_{\text {Dowell }}^{2}\left[1+\left(\frac{\lambda}{\sigma_{x}}\right)^{2} \cdot \frac{\delta}{3}\right],
$$

where $\delta=\left(p_{M}-p_{m}\right) / p_{m}$. Substitute a set of typical experimental values shown in Table I [7] into Eq. (11); it turns out to be 
TABLE I. Typical values of the physical parameters used in evaluation of Eq. (11).

\begin{tabular}{lcll}
\hline \hline Parameter & Value & Unit & \multicolumn{1}{c}{ Description } \\
\hline$E_{F}$ & 7.0 & $\mathrm{eV}$ & Fermi energy of copper \\
$\hbar \omega$ & 4.66 & $\mathrm{eV}$ & Photon energy \\
$\phi_{w}$ & 4.31 & $\mathrm{eV}$ & Work function of copper \\
$E_{\mathrm{rf}}$ & 50.0 & $\mathrm{MV} / \mathrm{m}$ & Applied electric field strength \\
$\lambda_{\text {opt }}$ & 12.58 & $\mathrm{~nm}$ & Mean-free path of the photon \\
$\bar{\lambda}_{\mathrm{e} \text { e }}$ & 6.13 & $\mathrm{~nm}$ & Mean-free path of the electron \\
\hline \hline
\end{tabular}

$$
\varepsilon_{D, x}^{2}=\varepsilon_{\text {Dowell }}^{2}\left[1+\left(\frac{0.40 \mathrm{~nm}}{\sigma_{x}}\right)^{2}\right] .
$$

The typical value of $\sigma_{x}$ is in the order of $100 \mu \mathrm{m}$; thus from the equation above, one could see it is totally acceptable to ignore the relations between the electron momentum distribution and the photon incident position. Dowell's formula is accurate in common cases.

\section{The approximate form of the emitted-electron phase-space distribution}

From Sec. II B one could say it is safe to ignore the dependence of electron momentum distribution on incident position. In fact this assertion could be proven directly from the PSF viewpoint without evaluating the statistical emittance in Eq. (8).

Let us consider the electron phase-space distribution at point $\mathbf{P}\left(x_{0}, y_{0}\right)$ on the surface. From PSF as shown in Eq. (6), it is easy to prove that the rms radius of the PSF is in the order of subnanometer and the PSF decays rapidly to zero in range out of the rms radius. Let us define the small circular area of which the center is point $\mathbf{P}$ and the radius is the PSF rms radius as "effective area." Since the effective area is very small, it is safe to assume that the laser intensity in the effective area will not change dramatically but keep almost constant. Also for the irradiated surface outside of the effective area, the contributions of the emitted electrons to point $\mathbf{P}$ are negligible. Following this idea, we could give an approximate form of the electron phase-space distribution at point $\mathbf{P}$ as Eq. (12):

$$
\begin{aligned}
\left.D\right|_{\mathbf{P}} & =\left.I(x, y) * f\left(x, y, p_{x}, p_{y}, p_{z}\right)\right|_{x=x_{0}, y=y_{0}} \\
& \left.\approx I\left(x_{0}, y_{0}\right) * f\left(x, y, p_{x}, p_{y}, p_{z}\right)\right|_{(x, y) \text { in effective area }} \\
& \left.\approx I\left(x_{0}, y_{0}\right) * f\left(x, y, p_{x}, p_{y}, p_{z}\right)\right|_{(x, y) \text { in entire area }} \\
& =I\left(x_{0}, y_{0}\right) \cdot \iint d x d y f\left(x, y, p_{x}, p_{y}, p_{z}\right) \\
& =I\left(x_{0}, y_{0}\right) f_{p}\left(p_{x}, p_{y}, p_{z}\right)
\end{aligned}
$$

which verified the assertion mentioned prior.

The $f_{p}\left(p_{x}, p_{y}, p_{z}\right)$ function in Eq. (12) is the momentum PSF, which describes the momentum-space distribution of the electrons that emitted at a single point. As shown in Eq. (12), $f_{p}$ is the integration of $f$ over the real-space $(x, y)$, which has the form of Eq. (13):

$$
\begin{aligned}
f_{p}\left(p_{x}, p_{y}, p_{z}\right)= & \frac{C_{p} p_{z}}{\sqrt{p_{z}^{2}+p_{m}^{2}} \cdot \sqrt{p_{x}{ }^{2}+p_{y}{ }^{2}+p_{z}{ }^{2}+p_{m}{ }^{2}}} \\
& \cdot H\left(p_{z}\right) H\left(p_{M}^{2}-p_{m}^{2}-p_{x}^{2}-p_{y}^{2}-p_{z}^{2}\right)
\end{aligned}
$$

where $C_{p}$ satisfies

$$
C_{p}=\frac{1-R}{1+\frac{\lambda_{\mathrm{opt}}}{\lambda_{\mathrm{e}-\mathrm{e}}}} \cdot \frac{1}{4 \pi m \hbar \omega} .
$$

The approximate form of the emitted electron distribution will play an important role in calculations of the roughness emittance in the following sections.

\section{SURFACE ROUGHNESS EMITTANCE OF 2D AND 3D SURFACE}

An unique advantage of PSF viewpoint is that it could be used to calculate the emittance of a roughness photocathode [19]. The surface of a real-life photocathode is not ideally smooth, but is weakly deformed. These weak deformations constitute the surface roughness. The surface topography of a real-life photocathode is shown in Fig. 2.

People usually use amplitude $a$ and spatial period $\lambda$ (or spatial wave number $k$ ) to describe a monochrome spatial component of the roughness. The roughness of a polished metallic photocathode could be roughly divided into two parts: the macroroughness $r_{M}$ and the microroughness

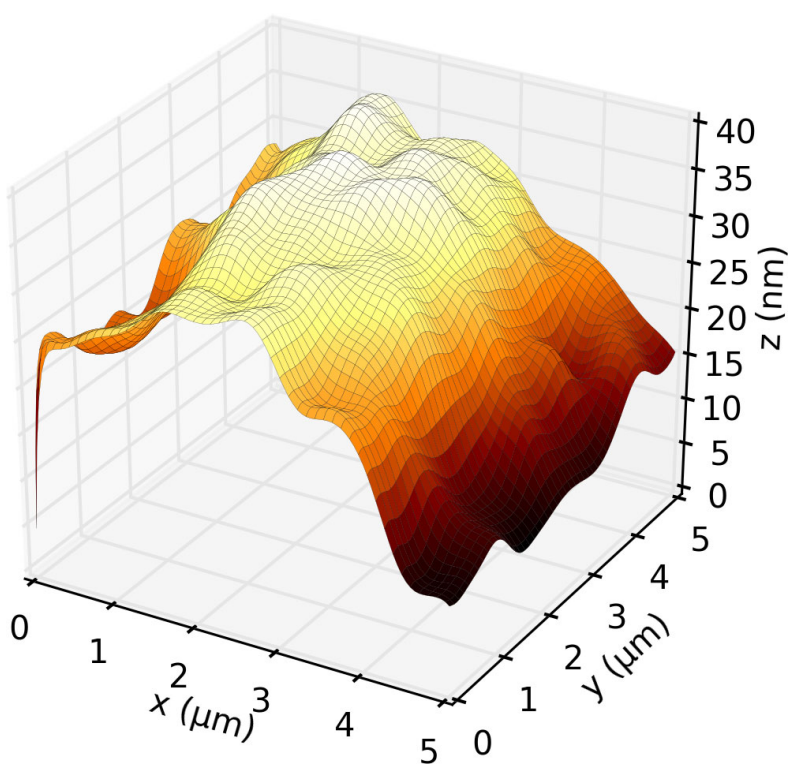

FIG. 2. Surface topography of part of a real-life photocathode. Note that the scales of $x$ axis ( $y$ axis) and $z$ axis are different. 
TABLE II. Typical values of the roughness parameters.

\begin{tabular}{lrll}
\hline \hline Parameter & Value & Unit & \multicolumn{1}{c}{ Description } \\
\hline$a_{m}$ & 4 & $\mathrm{~nm}$ & Amplitude of the microroughness \\
$\lambda_{m}$ & 200 & $\mathrm{~nm}$ & Period of the microroughness \\
$a_{M}$ & 100 & $\mathrm{~nm}$ & Amplitude of the macroroughness \\
$\lambda_{M}$ & 16 & $\mu \mathrm{m}$ & Period of the macroroughness \\
\hline \hline
\end{tabular}

$r_{m}$ [16]. The typical characteristics of the two kinds of roughness are shown in Table II, respectively.

It is obvious that the deformations of a well-polished cathode are rather weak; we could say the cathode has a "gently undulating surface." It is hard to rigorously define the gently undulating surface, since the concept differs from different situations. However in general, a gently undulating surface means that most of the slopes of the spatial frequency components of the surface should be much smaller than 1 . In this regard, we call a surface to be gently undulating in one direction (let us call it direction $x$ ) if

$$
\operatorname{rms}(R) \cdot \operatorname{rms}\left(k_{x}\right) \ll 1,
$$

where $k_{x}$ is the spatial wave number in direction $x, R$ is the amplitude of the considered surface.

The reasoning for this definition is to ensure that $k$. $R(x, y) \ll 1$ for $k \leq k_{\max }$; here $k_{\max }$ is the threshold of the spatial frequency components we are interested in. For the spatial frequency component of which $k>k_{\max }$, the amplitude of the component would be small enough to be irrelevant to roughness emittance growth. For instance, the spectrum of a real-life cathode surface (Fig. 10) is shown in Fig. 11. The statistic roughness properties calculated from the surface spectrum are: $\operatorname{rms}\left(k_{x}\right)=3.45 \mu \mathrm{m}^{-1}, \operatorname{rms}(R)=$ $22.65 \mathrm{~nm}$, thus $\operatorname{rms}(R) \cdot \operatorname{rms}\left(k_{x}\right)=0.08 \ll 1$. From the intensity distribution of the spectrum, we observe that the threshold of the spatial frequency we care about is $k_{x, \max } \sim 4 \mu \mathrm{m}^{-1}$ (since most of the spatial components are distributed within this threshold). For the sample surface above, one could see that $\operatorname{rms}\left(k_{x}\right) \sim k_{x, \max }$ and $R(x, y) \sim \operatorname{rms}(R)$, therefore our definition Eq. (14) could ensure that $k \cdot R(x, y) \ll 1$ for $k \leq k_{\max }$.

The following discussions and derivations are limited to this kind of surface.

\section{A. The generalized momentum PSF}

When a laser beam normally incident on a rough surface of which the surface morphology function is $R(x, y)$, we could track a single photon to see what happens, assuming that the photon injects into the cathode at point $\mathbf{P}\left(x_{0}, y_{0}\right)$. Since the normal vector of point $\mathbf{P}$ has an angle to the $z$ axis, the photoelectric process is almost the same as the smooth surface case except that the center of the emitted area is not at the incident point. The situation described above is shown in Fig. 3.

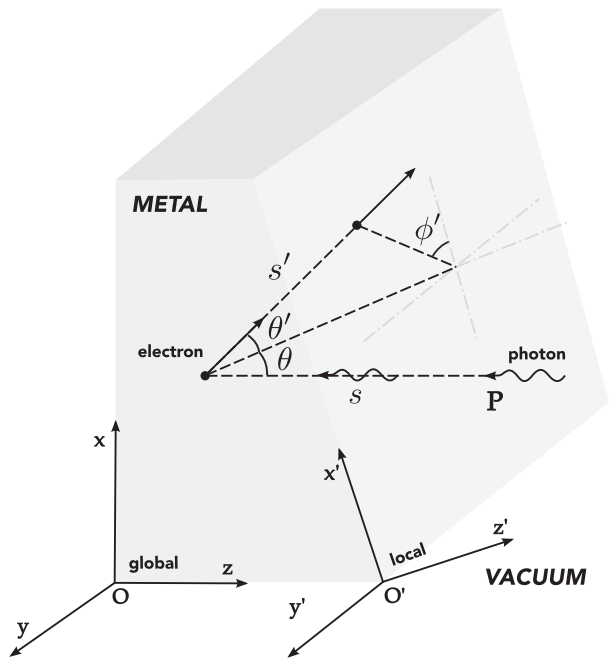

FIG. 3. The schematic plot for the bulk photoemission on a part of a rough metallic cathode. The definitions of coordinates and variables are marked in the plot. The main difference between the photoemission on a rough cathode and on a smooth cathode is that on the rough cathode, the photons are mostly not normal incident. As shown in the plot, a photon is injected into the surface with an incident angle $\theta$. After traveling along some distance $s$, the photon is absorbed by an electron, and then that electron travels towards the rough surface and emits from the cathode. It is obvious that the center of the emitted area is not point $\mathbf{P}$ anymore, it will have a small offset from point $\mathbf{P}$.

To consider the progress above in the PSF viewpoint, we could follow the same thought as explained in Sec. II C. The neighborhood $\Delta$ of point $\mathbf{P}$ could be treated as a plane of which the normal vector has an angle of $\theta$ to the $z$ axis, and the laser intensity is almost a constant in this small "effective" region, which is $I\left(x_{0}, y_{0}\right)$. To get the phasespace distribution of the emitted electrons in global coordinate $S$, one could first get the phase-space distribution in local coordinate $S^{\prime}$ of which the $z$ axis is normal to $\Delta$, then rotate the local distribution $D^{\prime}$ to get the global distribution $D$.

Intending to perform that, it is necessary to modify the original momentum PSF formation to make it applicable in the oblique incidence case (in local coordinate $S^{\prime}$, the laser is oblique incident). After some algebra, the generalized momentum PSF has the form of Eq. (15):

$$
\begin{aligned}
f_{p}\left(p_{x}, p_{y}, p_{z}\right) & =\frac{C_{p}(\theta) p_{z}}{\sqrt{p_{z}^{2}+p_{m}^{2}} \cdot \sqrt{p_{x}^{2}+p_{y}^{2}+p_{z}^{2}+p_{m}^{2}}}, \\
C_{p}(\theta) & =\frac{1-R(\theta)}{1+\frac{\lambda_{\mathrm{opt}}}{\lambda_{\mathrm{e} \cdot \mathrm{e}}} \cos \theta} \cdot \frac{1}{4 \pi m \hbar \omega} .
\end{aligned}
$$

To make the form clear we omitted the Heaviside functions. Using Eqs. (12) and (15), one could obtain the phase-space distribution in the local coordinate: 
$D^{\prime}\left(x, y, p_{x}, p_{y}, p_{z}\right)=I\left(x_{0}, y_{0}\right) \cos \theta f_{p}\left(p_{x}, p_{y}, p_{z}\right)$.

The next step is to rotate the local distribution to get the global distribution, which could be easily done by using the rotation matrix; the details are in Appendix C, here we only show the result in Eq. (17).

In fact, we do not really need Eq. (17) when calculating the roughness emittance, we just use Eq. (16) to calculate the local statistical terms in the neighborhood $\Delta$ of point
$\mathbf{P}\left(x_{0}, y_{0}\right)$, then use the rotation matrix to calculate the global emittance directly.

Now, the preparations are all done, we could move to the core: the calculation of the roughness emittance. For simplification, when deriving the global emittance in both the $2 \mathrm{D}$ and the $3 \mathrm{D}$ case, we will ignore the relation between weight $W(\theta)$ [defined as $W(\theta)=\cos \theta \cdot C_{p}(\theta)$, the weight is actually the effective $\mathrm{QE}]$ and $\theta$, the reason will be explained later in Sec. IV:

$$
\begin{aligned}
D & =I(x, y) \cdot \frac{\cos \theta \cdot C_{p}(\theta)\left(A p_{x}+B p_{y}+C p_{z}\right)}{\sqrt{\left(A p_{x}+B p_{y}+C p_{z}\right)^{2}+p_{m}^{2}}} \cdot \sqrt{p_{x}^{2}+p_{y}^{2}+p_{z}^{2}+p_{m}^{2}} \cdot H\left(p_{z}\right) H\left(p_{M}^{2}-p_{m}^{2}-p_{x}^{2}-p_{y}^{2}-p_{z}^{2}\right), \\
(A, B, C) & =\left(\frac{-\partial_{x} R}{\sqrt{1+\partial_{x}^{2} R+\partial_{y}^{2} R}}, \frac{-\partial_{y} R}{\sqrt{1+\partial_{x}^{2} R+\partial_{y}^{2} R}}, \frac{1}{\sqrt{1+\partial_{x}^{2} R+\partial_{y}^{2} R}}\right) .
\end{aligned}
$$

\section{B. 2D sinusoidal surface case}

First, let us consider a simple but heuristic 2D case. In this case, the surface morphology function is

$$
z=a \cos k x
$$

and the coordinate system is shown in Fig. 4 .

As explained in Sec. III A, consider an incident point at position $\mathbf{P}$, assuming that under the global coordinate the transverse and longitudinal momentum of one electron emitted from $\mathbf{P}$ are $p_{x}, p_{z}$, respectively. Applying the $2 \mathrm{D}$ rotation matrix, we get

$$
\left(\begin{array}{c}
p_{x} \\
p_{z}
\end{array}\right)=\left(\begin{array}{cc}
\cos \theta & -\sin \theta \\
\sin \theta & \cos \theta
\end{array}\right)\left(\begin{array}{c}
p_{x}^{\prime} \\
p_{z}^{\prime}
\end{array}\right)
$$

The angle $\theta$ is the angle from the $z$ axis to the normal vector of point $\mathbf{P}$, and $p_{x}^{\prime}, p_{z}^{\prime}$ are the transverse and longitudinal momentum of the electron under local coordinate, respectively. The above equation shows how the emission angle diffusion affects the emitted electron transverse momentum, thus causing thermal emittance growth.

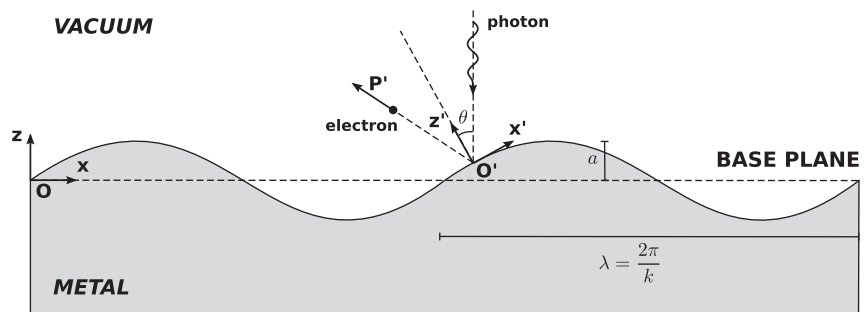

FIG. 4. The schematic plot for the bulk photoemission on a 2D sinusoidal surface. The definitions of coordinates and variables are marked in the plot.
Now let us consider the effect of the transverse applied electric field. First, we need to know the approximate form of the transverse field. Define that $\xi=a k$, from [20], if $\xi$ is rather small, the electric field near the deformed surface could be written as follows:

$$
\begin{aligned}
& E_{x}=E \xi \cdot e^{-k z} \sin k x, \\
& E_{z}=E\left(1+\xi e^{-k z} \cos k x\right) .
\end{aligned}
$$

Letting $A=e E / m$, it could be shown that the transverse momentum of the electron approximatively satisfies the following equation:

$$
p_{\infty}-p_{0}=m \sqrt{\frac{\pi A}{2 k}} \xi \sin k x,
$$

where $p_{\infty}$ is the final momentum of an electron which experienced the transverse electric field near the surface.

Adding the two effects together, we obtain the final $p_{x}$ formation:

$$
p_{x}=p_{x}^{\prime} \cos \theta-p_{z}^{\prime} \sin \theta+m \sqrt{\frac{\pi A}{2 k}} \xi \sin k x
$$

in which

$$
\begin{aligned}
& \cos \theta=\frac{1}{\sqrt{1+\xi^{2} \sin ^{2} k x}} \approx 1-\frac{1}{2} \xi^{2} \sin ^{2} k x, \\
& \sin \theta=\frac{-\xi \sin k x}{\sqrt{1+\xi^{2} \sin ^{2} k x}} \approx-\xi \sin k x .
\end{aligned}
$$

Substituting all the above into Eq. (8), letting $p_{C}=$ $m \sqrt{\pi A / 2 k}$ ( $p_{C}$ has the dimension of momentum), we get 
the final form of the roughness emittance as shown in Eq. (18). Here we define the square root of the term in the square brackets of Eq. (18) as emittance growth factor $\eta$, so that Eq. (18) could be rewritten in the form of $\varepsilon_{x}=\eta \cdot \varepsilon_{D, x}$ :

$$
\varepsilon_{x}^{2}=\varepsilon_{D, x}^{2}\left[1+\frac{1}{2} \xi^{2}\left(\frac{\left\langle\left(p_{z}^{\prime}+p_{C}\right)^{2}\right\rangle}{\left\langle p_{x}^{\prime 2}\right\rangle}-1\right)\right] .
$$

When the applied field strength is zero, Eq. (18) degenerates into the slope effect roughness emittance Eq. (19) [15]; when $p_{z}^{\prime} \ll p_{C}$, one could ignore the $p_{z}^{\prime}$ term and thus Eq. (18) degenerates into the field effect roughness emittance Eq. (20) [16]:

$$
\begin{gathered}
\varepsilon_{x}^{2} \approx \varepsilon_{D, x}^{2}\left(1+\frac{1}{2} \xi^{2}\right), \\
\varepsilon_{x}^{2} \approx \varepsilon_{D, x}^{2}\left(1+\frac{3 \pi e}{4} \cdot \frac{a^{2} k E}{\hbar \omega-\phi_{\mathrm{eff}}}\right) .
\end{gathered}
$$

Substituting the typical values of microroughness and macroroughness parameters (see Table II) into Eqs. (18)(20), one could see that for the microroughness, the emittance growth factor is around 1.08, while for the macroroughness, the emittance growth factor is about 1.35 , which shows an evidence that the emittance growth mainly due to the macroroughness. Keeping $\xi$ or $a$ as a constant, one could explore the relation between the spatial period $\lambda$ of the roughness and the emittance growth factor $\eta$ as shown in Fig. 5.

Just as the solid curve in the left subplot of Fig. 5 shows, the emittance growth factor and the spatial period of the surface pattern are positively correlated while rescaling the sinusoidal surface, which verifies that the main contribution to the roughness emittance is from the macroroughness.

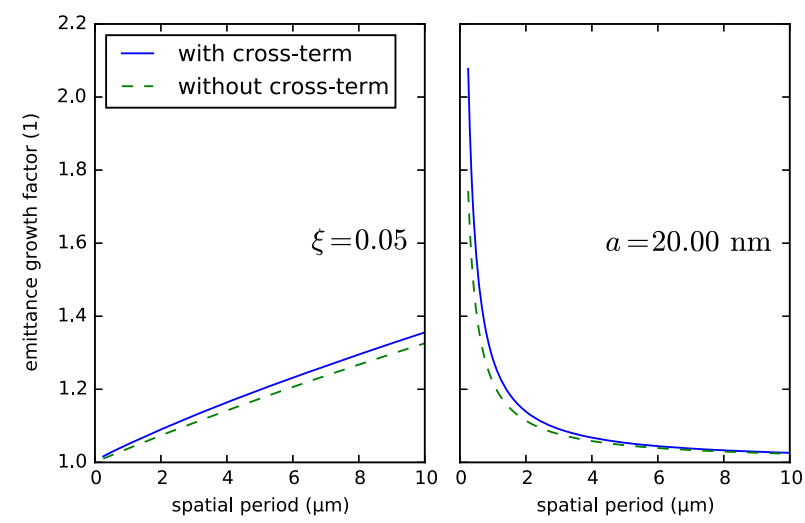

FIG. 5. Emittance growth factor vs spatial period for a 2D sinusoidal surface. The applied field strength is $50 \mathrm{MV} / \mathrm{m}$. The subplot on the left: Keep aspect (amplitude over spatial period) of the pattern on the surface as a constant $(\xi=0.05)$. The subplot on the right: Keep the amplitude of the surface as a constant $(a=20.00 \mathrm{~nm})$.

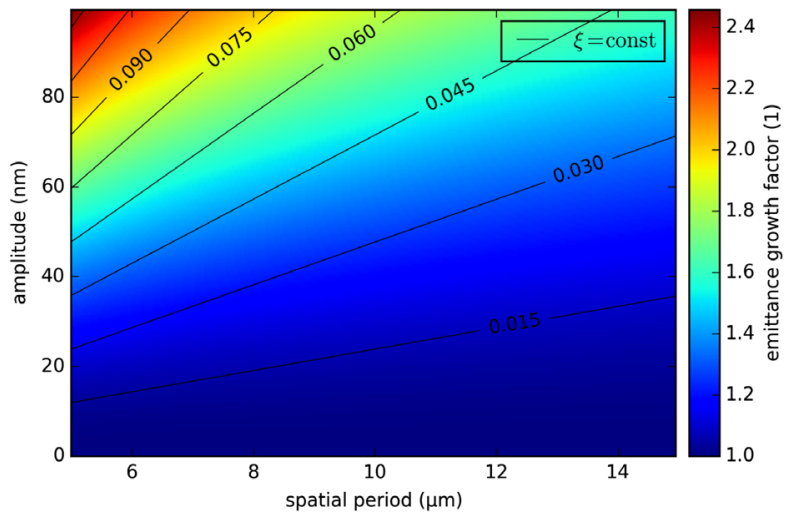

FIG. 6. Contour plot of the emittance growth factor. Note that the solid black lines in the plot stand for different aspect $(\xi)$ of the surface, but not for contour lines for the emittance growth factor.

The dashed curve in Fig. 5 stands for Eq. (18), which ignores the cross term when expanding Eq. (18). It is easy to observe that there is a certain amount of difference between the solid and dashed curves. Detailed calculation shows that the relative error of the emittance growth could be as high as $10 \%$, which indicates that it is inappropriate to ignore the cross term between the intrinsic emittance and the roughness emittance.

From Fig. 5 we could conclude two things: (i) The smoother the undulation of the surface is, the smaller the emittance growth will be; (ii) while keeping the shape of the surface roughness the same, the larger the scale is, the bigger the emittance growth will be. A more complete understanding among the spatial period, the amplitude and the emittance growth factor is revealed in Fig. 6.

\section{2D random surface case}

The coordinate system for the $2 \mathrm{D}$ random surface case is shown in Fig. 7.

The idea to calculate $2 \mathrm{D}$ random surface roughness emittance is the same as the one used in Sec. III B. The emission angle diffusion related momentum growth calculation part is exactly the same, so we skip that part and pass to the transverse field related part directly.

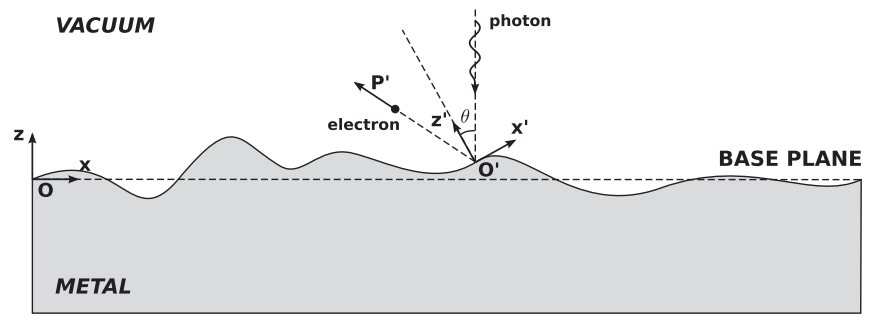

FIG. 7. The schematic plot for the bulk photoemission on a 2D random surface. The definitions of coordinates and variables are marked in the plot. 
Let us assume that the surface morphology function is

$$
z=R(x)
$$

We choose the base plane so that $\langle R(x)\rangle=0$. As always, in order to evaluate the field related transverse momentum growth, we need to get the formation of the transverse field. Inspired by [20], one could assume the electric potential of the space between the cathode surface $z=R(x)$ and infinity $z=+\infty$ has the approximate form:

$$
\phi(x, z)=z+\int d k C(k) \cdot e^{j k x-|k| z}
$$

which naturally satisfies Laplace's equation $\Delta \phi=0$ and boundary condition at $z=\infty:\left.\phi\right|_{z=d}=d, d \rightarrow \infty$ (unit electric field strength). Now let us consider the boundary condition at $z=R(x), \phi$ must satisfy that

$$
\left.\phi(x, z)\right|_{z=R(x)}=R(x)+\int d k C(k) \cdot e^{j k x-|k| R(x)} \equiv 0 .
$$

Since the surface is a gently undulating surface as defined in Eq. (14), $|k| R(x)$ should be a first order quantity. Expand $e^{-|k| R(x)}$ as $1-|k| R(x)$, substituting into the equation above, one could obtain that

$$
R(x)+\int d k C(k) \cdot e^{j k x}=O(1) \approx 0 .
$$

Performing Fourier transformation on $R(x)$,

$$
R(x)=\int d k R(k) \cdot e^{j k x}
$$

By comparing each term it is easy to see that $C(k)=-R(k)$, therefore we have the first order approximate form of $\phi$ as the following:

$$
\phi(x, z)=z-\int d k R(k) \cdot e^{j k x-|k| z} .
$$

To verify the approximation made above, we choose one random gently undulating $2 \mathrm{D}$ surface, and draw both the surface morphology and the approximate potential map. Setting the electric potential at the surface to be zero, then the closer the zero-potential surface of approximate potential map to the random surface morphology, the more accurate the approximation would be. The comparison is shown as Fig. 8.

From Fig. 8 one could safely believe that the approximate form of $2 \mathrm{D}$ potential is accurate enough. Applying $\boldsymbol{E}=-\nabla \phi$, one gets

$$
\begin{aligned}
& E_{x}=j \int d k \cdot k R(k) \cdot e^{j k x-|k| z}, \\
& E_{z}=-1-\int d k \cdot|k| R(k) \cdot e^{j k x-|k| z} .
\end{aligned}
$$

Now assuming that the electric field strength is $E$, using the Newton's second law, one could obtain that

$$
\begin{aligned}
p_{\infty}-p_{0} & =m \sqrt{\frac{A}{2}} \cdot \int-\frac{E_{x}}{\sqrt{z}} d z \\
& =-j m \sqrt{\frac{\pi A}{2}} \cdot \int d k \frac{k}{\sqrt{|k|}} R(k) \cdot e^{j k x} .
\end{aligned}
$$

Finally the emittance could be written as

$$
\begin{aligned}
\varepsilon_{x}^{2}= & \varepsilon_{D, x}^{2}\left[1-\left\langle R^{\prime 2}\right\rangle\right. \\
& \left.+\frac{\left\langle\left(p_{z}^{\prime} \cdot R^{\prime}+j m \sqrt{\frac{\pi A}{2}} \cdot \int d k \frac{k}{\sqrt{|k|}} R(k) \cdot e^{j k x}\right)^{2}\right\rangle}{\left\langle p_{x}^{\prime 2}\right\rangle}\right] .
\end{aligned}
$$

To verify the correctness of Eq. (22), we consider a special case that was discussed in Sec. III B, where the

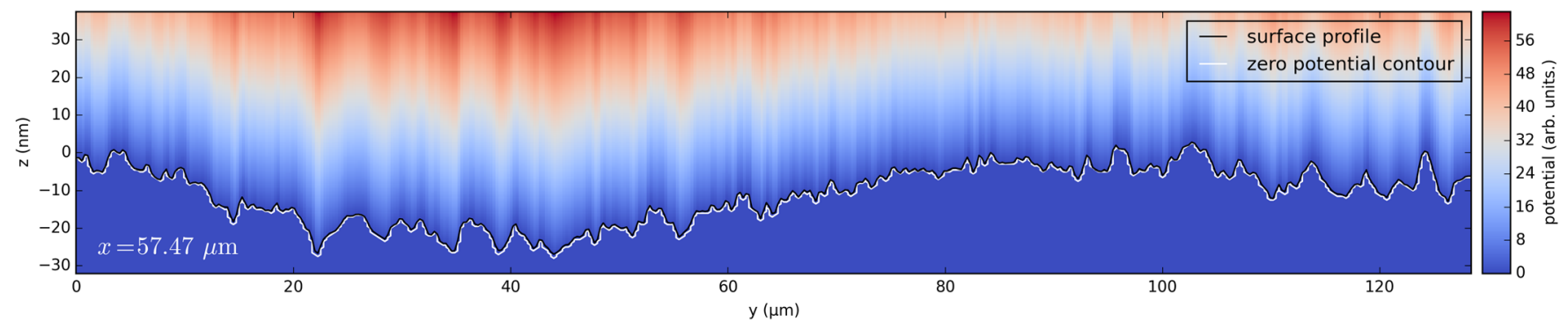

FIG. 8. Validation of the accuracy of the analytical electric potential. We take the profile of the 3D surface shown in Fig. 10 along $x=57.47 \mu \mathrm{m}$, the surface profile is marked by the black bold curve. The white bold curve in the plot is the zero potential contour of the analytical electric potential in the $y$ - $z$ plane at $x=57.47 \mu \mathrm{m}$, which is calculated by the equations. In the plot, the surface profile and the zero potential contour are mostly overlapped, therefore we conclude that the analytical electric potential is quite accurate. 
surface morphology function is $R(x)=a \cos k_{0} x$ (assuming that $k_{0}>0$ ). Let

$$
I(x)=j m \sqrt{\frac{\pi A}{2}} \cdot \int d k \frac{k}{\sqrt{|k|}} R(k) \cdot e^{j k x}
$$

then $R(k)=\frac{a}{2}\left[\delta\left(k-k_{0}\right)+\delta\left(k+k_{0}\right)\right]$; therefore

$$
\begin{aligned}
I(x) & =j m \sqrt{\frac{\pi A}{2}}\left[\frac{a}{2} \frac{k_{0}}{\sqrt{\left|k_{0}\right|}} e^{j k_{0} x}-\frac{a}{2} \frac{k_{0}}{\sqrt{\left|k_{0}\right|}} e^{-j k_{0} x}\right] \\
& =m \sqrt{\frac{\pi A}{2 k_{0}}} \cdot\left(-a k_{0} \sin k_{0} x\right)=p_{C} \cdot R^{\prime}
\end{aligned}
$$

thus

$$
\begin{aligned}
\varepsilon_{x}^{2} & =\varepsilon_{D, x}^{2}\left[1-\left\langle R^{\prime 2}\right\rangle+\frac{\left\langle\left(p_{z}^{\prime} \cdot R^{\prime}+I(x)\right)^{2}\right\rangle}{\left\langle p_{x}^{\prime 2}\right\rangle}\right] \\
& =\varepsilon_{D, x}^{2}\left[1+\left\langle R^{\prime 2}\right\rangle\left(\frac{\left\langle\left(p_{z}^{\prime}+p_{C}\right)^{2}\right\rangle}{\left\langle p_{x}^{\prime 2}\right\rangle}-1\right)\right] \\
& =\varepsilon_{D, x}^{2}\left[1+\frac{1}{2} \xi^{2}\left(\frac{\left\langle\left(p_{z}^{\prime}+p_{C}\right)^{2}\right\rangle}{\left\langle p_{x}^{\prime 2}\right\rangle}-1\right)\right]
\end{aligned}
$$

which is identical to Eq. (18). This identity shows that for a special 2D sinusoidal surface, the formula Eq. (22) degenerates into Eq. (18), which is a trustworthy result. Therefore the general formula for 2D surface Eq. (22) is reliable.

Just to mention, $I(x)$ could be written as an alternative form:

$$
I(x)=\frac{m}{2} \sqrt{\frac{A}{|x|}} * R^{\prime}(x)
$$

by applying the convolution theory, and knowing that

$$
\mathcal{F}\left(\frac{1}{\sqrt{|x|}}\right)=\frac{\sqrt{2 \pi}}{|k|}
$$

where $\mathcal{F}(f(x))=\int f(x) e^{-j k x} d x$ is the Fourier transform of $f(x)$, one could easily get Eq. (23).

\section{3D random surface case}

Using the same idea as described in Sec. III C, one could easily solve the 3D case emittance calculation problem.
Assume that the 3D surface morphology function is

$$
z=R(x, y)
$$

We choose the base plane so that $\langle R(x, y)\rangle=0$. We suppose the form of the electric potential of the space between the cathode surface $z=R(x, y)$ and infinity $z=$ $+\infty$ has the approximate form:

$$
\phi(x, y, z)=z+\int d k_{x} d k_{y} C\left(k_{x}, k_{y}\right) \cdot e^{j\left(k_{x} x+k_{y} y\right)-k z},
$$

where $k=\sqrt{k_{x}^{2}+k_{y}^{2}}$. It is easy to prove that the form above satisfies the Laplace's equation. By using the boundary condition on the conductor surface, one could get that $C\left(k_{x}, k_{y}\right)=-R\left(k_{x}, k_{y}\right)$ where $R\left(k_{x}, k_{y}\right)$ is the coefficient of Fourier transformation of $R(x, y)$. So the electric potential could be written as

$$
\phi(x, y, z)=z-\int d k_{x} d k_{y} R\left(k_{x}, k_{y}\right) \cdot e^{j\left(k_{x} x+k_{y} y\right)-k z} .
$$

Thus the electric field has the form

$$
\begin{aligned}
& E_{x}=j \int d k_{x} d k_{y} \cdot k_{x} R\left(k_{x}, k_{y}\right) \cdot e^{j\left(k_{x} x+k_{y} y\right)-k z}, \\
& E_{y}=j \int d k_{x} d k_{y} \cdot k_{y} R\left(k_{x}, k_{y}\right) \cdot e^{j\left(k_{x} x+k_{y} y\right)-k z}, \\
& E_{z}=-1-\int d k_{x} d k_{y} \cdot k R\left(k_{x}, k_{y}\right) \cdot e^{j\left(k_{x} x+k_{y} y\right)-k z} .
\end{aligned}
$$

Now considering the transverse dynamics of emitted electrons we get

$$
\begin{aligned}
p_{\infty}-p_{0} & =m \sqrt{\frac{A}{2}} \cdot \int-\frac{E_{x}}{\sqrt{z}} d z \\
& =-j m \sqrt{\frac{\pi A}{2}} \cdot \int d k_{x} d k_{y} \frac{k_{x}}{\sqrt{k}} R\left(k_{x}, k_{y}\right) \cdot e^{j\left(k_{x} x+k_{y} y\right)} .
\end{aligned}
$$

Following the same steps of Sec. III C, it is not difficult to derive the equation of roughness emittance as shown in Eq. (25):

$$
\varepsilon_{x}^{2}=\varepsilon_{D, x}^{2}\left[1-\left\langle\partial_{x}^{2} R\right\rangle+\frac{\left\langle\left(p_{z}^{\prime} \cdot \partial_{x} R+j m \sqrt{\frac{\pi A}{2}} \cdot \int d k_{x} d k_{y} \frac{k_{x}}{\sqrt{k}} R\left(k_{x}, k_{y}\right) \cdot e^{j\left(k_{x} x+k_{y} y\right)}\right)^{2}\right\rangle}{\left\langle p_{x}^{\prime 2}\right\rangle}\right] .
$$

Similar to the 2D case, substituting E in Eq. (25) with zero, one could obtain the emission angle diffusion related roughness emittance Eq. (26): 


$$
\varepsilon_{x}^{2}=\varepsilon_{D, x}^{2}\left(1+\left\langle\partial_{x}^{2} R\right\rangle\right) .
$$

Using Fourier energy conservation relation, the $\left\langle\partial_{x}^{2} R\right\rangle$ term could be written as the following form:

$$
\left\langle\partial_{x}^{2} R\right\rangle=\frac{\iint_{F(S)} k_{x}^{2}\left|R\left(k_{x}, k_{y}\right)\right|^{2} d k_{x} d k_{y}}{\iint_{S} d x d y} .
$$

Note that the product of spatial harmonic amplitude $\left|R\left(k_{x}, k_{y}\right)\right|$ and spatial harmonic wave number $k_{x}$ gives the $\xi$ parameter that decides the amount of the slope effect roughness emittance in the $2 \mathrm{D}$ sinusoidal case, which means that the results of the $2 \mathrm{D}$ and the $3 \mathrm{D}$ case are in consistence, and agree with others' results [15].

Though the form for the 3D random surface roughness emittance is not as simple as the 2D sinusoidal surface one, we could still get some meaningful information from Eq. (25). For example, let us focus on the relations between the emittance growth factor $\eta$ and the strength of the applied field $E$, expanding Eq. (25) as function of $E$ would give

$$
\eta^{2}=a E+b \sqrt{E}+c
$$

in which $a, b$ and $c$ are related to the surface morphology. Some experimental studies have shown the similar relationship between $E$ and $\varepsilon$ [10].

\section{NUMERICAL SIMULATIONS}

While calculating the emittance, we ignored the relation between $W(\theta)$ and $\theta$, as mentioned in Sec. III A. Here we will give the reason. The reflectivity $R(\theta)$ and weight $W(\theta)$ with relation of the incident angle $\theta$ are shown in Fig. 9. The inset of Fig. 9 is a zoom-in of the weight curve between $0 \mathrm{deg}$ and $1 \mathrm{deg}$. It is easy to see that for incident angle smaller than $1 \mathrm{deg}$, the weight is almost constant. That is

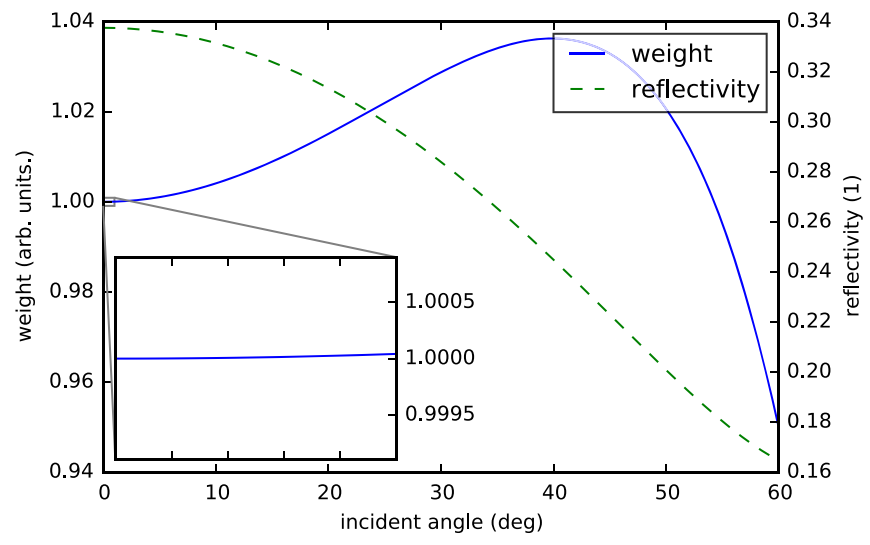

FIG. 9. Weight $W(\theta)$ and reflectivity $R(\theta)$ vs incident angle $\theta$ for a smooth copper photocathode. $W(\theta)$ is actually the effective $\mathrm{QE}$, which is proportional to the electron emission intensity on the cathode. The inset is a zoom-in of the weight curve between $0 \mathrm{deg}$ and $1 \mathrm{deg}$.

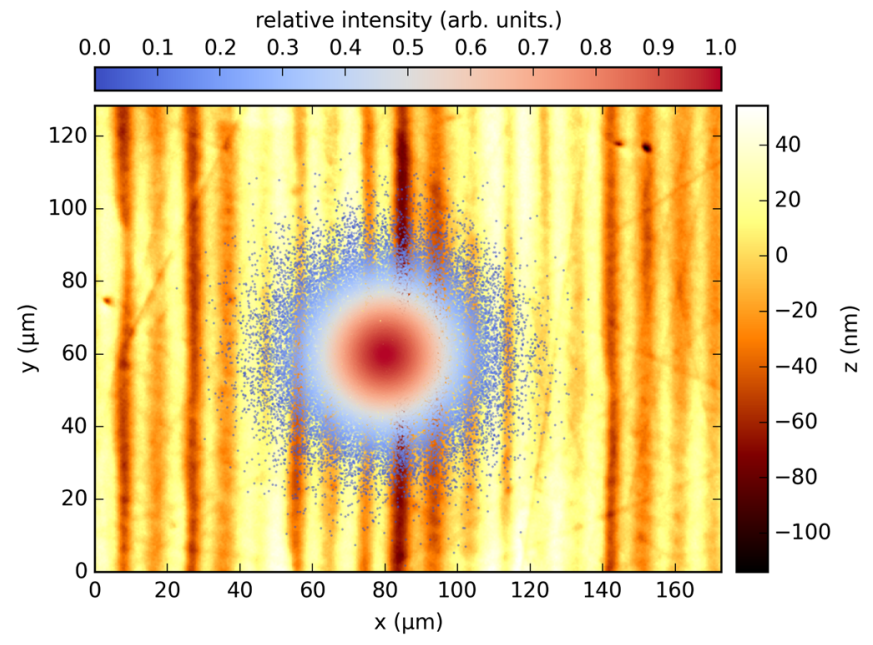

FIG. 10. Simulation configuration. The yellow-black background shows the morphology of the surface we employed in the simulation, while the blue-red spot in the center describes the transverse intensity distribution of the laser.

why we could treat $W(\theta)$ as $\theta$-independent in generalized PSF Eq. (15). We will consider $W(\theta)$ as a constant during the whole numerical simulation.

\section{A. Simulation configuration}

Our simulation configuration is shown in Fig. 10, the blue-red area near the center is the laser incident zone. The background is surface morphology of a real cathode (based on data from white light interferometer), of which the roughness spectrum (the Fourier transform of the surface) is shown in Fig. 11. We set the material of the cathode to be copper (the material would influence the work function, the

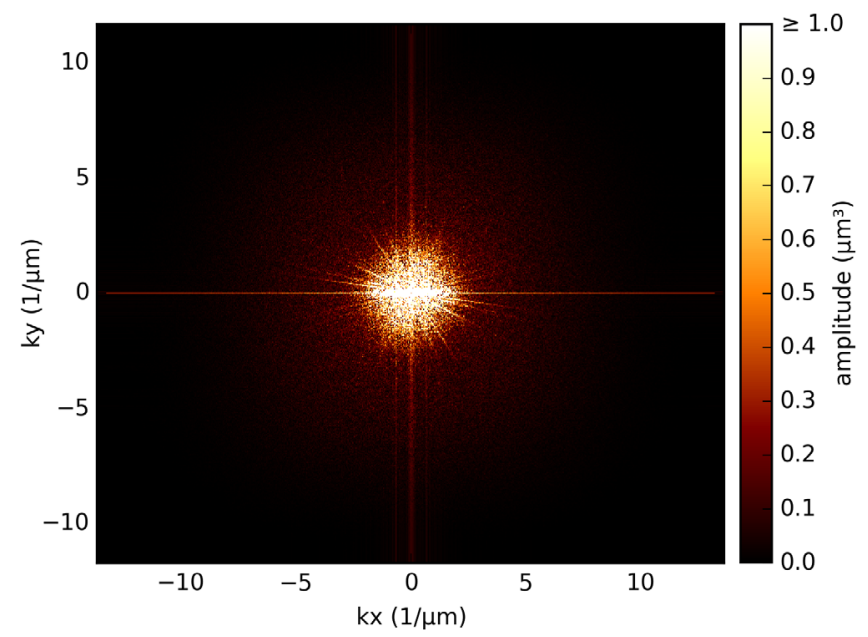

FIG. 11. The Fourier transform of the surface employed in the simulation. Note that in order to make high-order components visible, we set the threshold amplitude to be $1 \mu \mathrm{m}^{3}$, which means that the components with amplitudes larger than $1 \mu \mathrm{m}^{3}$ would be plotted as if their amplitudes are $1 \mu \mathrm{m}^{3}$. 
mean-free path of electron and photon which involves QE and emittance calculation, etc). To take account of the field effect, we also employ a high voltage on the surface, for simplification the high voltage will not change with time, which is reasonable considering that during the short laser pulse, the phase of the rf field would not change significantly.

Under this configuration, we will perform several dynamics simulations on the emitted electron bunch, then do statistics to get the initial and saturated emittance, finally figure out the emittance growth factor under different conditions.

\section{B. Principles of the simulation}

To simulate the effect due to the surface roughness, we need a rough surface, an applied electric field that would produce transverse electric field on the rough surface, and a laser. We generate the rough surface by using the surface morphology data from a real cathode, and produce the electric field map by applying the related formulas from Sec. III D on the rough surface. To generate the initial electron beam that emitted from the surface when irradiated by a laser, first we generate a sequence of positions that obeys the transverse distribution of the laser, then for each position, generate the momentum that satisfies the momentum PSF at that position (different positions on the surface will have different normals, thus their PSF are slightly different), finally combine the position sequence and the momentum sequence would give the initial electron samples.

We employ the 5th order Runge-Kutta method to do the motion equation integration. For technical reasons, we prefer using the distance from the surface base plane $z$ as the variable rather than time $t$. The electron motion equation about $z$ could be written as

$$
\begin{aligned}
\frac{d p_{x}[\mathrm{keV} / \mathrm{c}]}{d z[\mathrm{~nm}]} & =511 \times 10^{-6} \cdot \frac{E_{0}[\mathrm{MV} / \mathrm{m}]}{p_{z}[\mathrm{keV} / \mathrm{c}]} \cdot \hat{E}_{x}(x, y, z), \\
\frac{d x[\mu \mathrm{m}]}{d z[\mathrm{~nm}]} & =\frac{p_{x}[\mathrm{keV} / \mathrm{c}]}{p_{z}[\mathrm{keV} / \mathrm{c}]} \cdot 1 \times 10^{-3},
\end{aligned}
$$

where $x$ stands for both $x$ and $y$ direction, $E_{0}$ is the electric field strength, and $\hat{E}_{x}$ is the transverse normalized electric field distribution. Notice that for convenience, we use $\mu \mathrm{m}$ as the length unit for transverse direction but $\mathrm{nm}$ for longitudinal direction.

Knowing that the transverse components of the electric field will vanish as the distance to the surface base plane goes up, the transverse momentum of the emitted electron would be saturated at a large $z$ (typically around $5000 \mathrm{~nm}$ ). We do statistics at that position to get the emittance growth factor.

We wrote a PYTHON code to realize all things mentioned above.
TABLE III. Parameters used in numerical simulation.

\begin{tabular}{llll}
\hline \hline Parameter & Value & Unit & \multicolumn{1}{c}{ Description } \\
\hline$\lambda_{l}$ & 266.0 & $\mathrm{~nm}$ & Laser wavelength \\
1-dist & uniform & $\ldots$ & laser transverse distribution \\
$r_{l}$ & 20.0 & $\mu \mathrm{m}$ & Laser transverse radius \\
$x_{l}$ & 80.0 & $\mu \mathrm{m}$ & Laser incident x center \\
$y_{l}$ & 60.0 & $\mu \mathrm{m}$ & Laser incident y center \\
mat & Copper & $\ldots$ & Material of the cathode \\
$E_{0}$ & 50.0 & $\mathrm{MV} / \mathrm{m}$ & Effective electric field strength \\
$\phi_{w}$ & 4.31 & $\mathrm{eV}$ & Work function \\
$\phi_{\text {eff }}$ & 4.04 & $\mathrm{eV}$ & Effective work function \\
$E_{F}$ & 7.0 & $\mathrm{eV}$ & Fermi energy \\
$N$ & 10000 & $\cdots$ & Number of particles \\
$z_{i}$ & 0 & $\mathrm{~nm}$ & Simulation starting position \\
$z_{f}$ & 5000.0 & $\mathrm{~nm}$ & Simulation ending position \\
$d z$ & 10.0 & $\mathrm{~nm}$ & Simulation $z$ step \\
\hline \hline
\end{tabular}

\section{Simulation results}

Using the parameters in Table III, we get results shown in Fig. 12.

From Fig. 12 one could see that the phase space is distorted along the $x$ direction. The distortion is caused by the transverse electric field on the surface, and this distortion enables the emittance growth. Doing statistics on both the initial phase space and the final one, we obtain that the emittance growth factor is

$$
\eta_{s}=\frac{\varepsilon_{f}}{\varepsilon_{i}}=\frac{4.826 \mu \mathrm{m} \cdot \mathrm{keV} / \mathrm{c}}{4.623 \mu \mathrm{m} \cdot \mathrm{keV} / \mathrm{c}}=1.044
$$
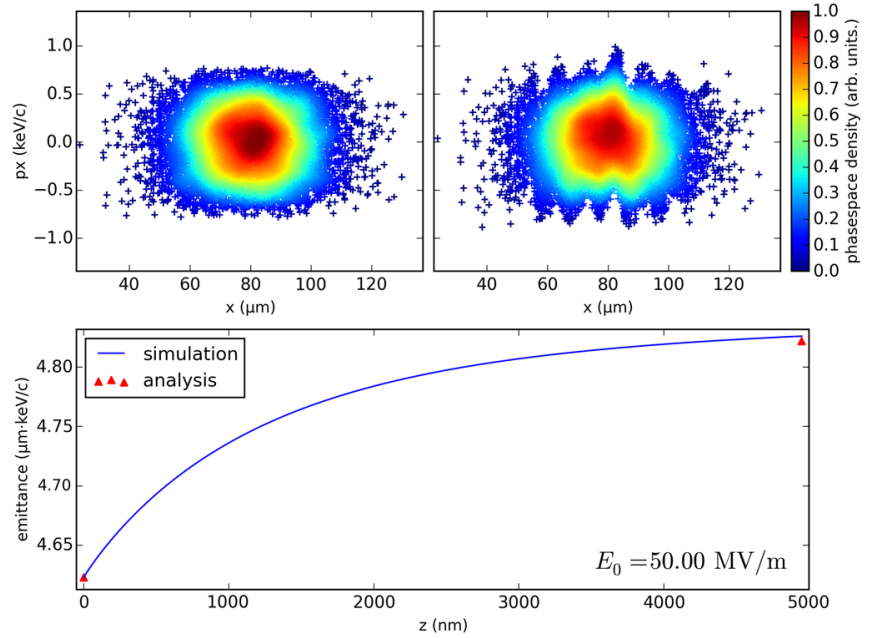

FIG. 12. Simulated evolution of the horizontal ( $x$ direction) phase space and emittance of the electron beam generated from the rough copper surface (Fig. 10). The upper left subplot is the initial phase space at $z=0 \mathrm{~nm}$, the upper right subplot is the final phase space at $z=5000 \mathrm{~nm}$, and the lower subplot is the evolution of $\varepsilon_{x}$ along $z$. The two red triangles in the lower subplot are the initial analytical emittance and final analytical emittance, respectively. 
Surprisingly the emittance growth factor is far smaller than expected (1.5-2). However some experiments give that the emittance growth factor should be around 1.5 to 2, comparing with our simulation result, it implies that the surface roughness effect-including both the slope effect and the field effect-might not be the only reason for the emittance growth that was observed in experiments.

\section{Comparison with the analytical result}

We compared the simulation emittance result with the analytical result calculated by Eq. (25) in Fig. 12. The red triangles stand for the analytical results: the left one stands for emittance with emission angle discrete effect $\left(\varepsilon_{1}\right)$, and the right one is the total emittance that includes the transverse electric field effect $\left(\varepsilon_{2}\right)$. Since when we generate the samples, we already include the emission angle discrete effect, and we use the same samples for simulation and analytical calculation, thus $\varepsilon_{1}$ from simulation and analytical result should be identical.

For $\varepsilon_{2}$, the simulation one should be a little bit smaller but asymptotically equal to the theoretical result, however Fig. 12 shows the opposite. The possible reason is our simulation step size is too large, since the transverse electric field will decrease with distance $z$, the motion equation integration will be larger than the real one (because the accumulation at each step will use the old field, which is larger than the average field).

Even though, since the emittance growth factor that was given by the analytical formula is

$$
\eta_{a}=\frac{\varepsilon_{2}}{\varepsilon_{1}}=\frac{4.822 \mu \mathrm{m} \cdot \mathrm{keV} / \mathrm{c}}{4.623 \mu \mathrm{m} \cdot \mathrm{keV} / \mathrm{c}}=1.043
$$

the simulation and analytical values are rather close, thus we could confidently say the analytical result is reliable.

By using the analytical formula, the calculation of the emittance growth factor will be speeded up significantly compared to the simulation routine, which enables the possibility to explore on the relation between electric field strength and emittance growth factor. The result is shown in Fig. 13 (the solid blue curve). It tells us the emittance growth caused by 3D surface roughness is rather small, even in the case that applys a $120 \mathrm{MV} / \mathrm{m}$ electric field, the growth factor is still smaller than 1.1.

In Fig. 13, we also compared the emittance growth factor between several $2 \mathrm{D}$ sinusoidal surface cases and the 3D random surface case. We choose different sets of spatial period ( $p$ in the legend) and amplitude $a$ for 2D sinusoidal cases. The details are described in the caption. It is obvious that the emittance growth factor for the 2D macroroughness case (dotted magenta curve) is significantly larger than the 3D case under the same applied electric field strength. However, the comparison would be meaningless if the roughness parameters of the $2 \mathrm{D}$ surface and the $3 \mathrm{D}$ surface are not matched. Therefore we performed the roughness

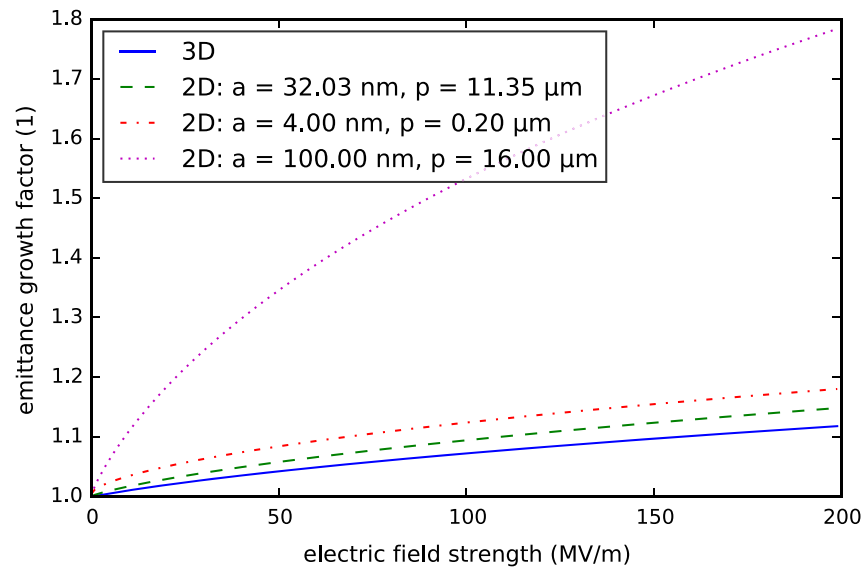

FIG. 13. Comparison between 2D and 3D emittance growth factor. The solid blue curve is calculated from the 3D rough surface shown in Fig. 10. The dashed green curve is calculated from the roughness parameters which are matched to the $3 \mathrm{D}$ rough surface. The dash-dotted red curve and the dotted magenta curve are calculated from the microroughness and macroroughness parameters from Table II, respectively.

statistic on the 3D surface and found that the rms roughness $R_{q}$ and rms average wavelength $\lambda_{q}$ [21] to be $22.65 \mathrm{~nm}$ and $11.35 \mu \mathrm{m}$, respectively. The parameters for the matched 2D surface satisfy that

$$
\begin{aligned}
a & =\sqrt{2} R_{q}, \\
\lambda & =\lambda_{q} .
\end{aligned}
$$

We then got $a=32.03 \mathrm{~nm}$ and $\lambda=11.35 \mu \mathrm{m}$ from the equations above. Applying the parameters obtained, we plot the emittance growth factor curve for the matched 2D surface as shown by the dashed green curve in Fig. 13. This time the emittance growth factor curves for the 3D and the $2 \mathrm{D}$ case are very close, with the fact that the $3 \mathrm{D}$ curve is a little lower than the $2 \mathrm{D}$ one. The possible reason for the difference might be that the transverse momentum growth due to the 3D surface roughness would spread out in the whole $x-y$ plane, while for the $2 \mathrm{D}$ case the transverse momentum growth would be sustained in the $x$ direction, thus with the same statistic roughness properties, the 2D emittance growth would be larger than the $3 \mathrm{D}$ case.

The propinquity of the emittance growth between the matched $2 \mathrm{D}$ and $3 \mathrm{D}$ surface gives us a simple way to estimate the upper limit of the roughness emittance growth on a real cathode: first get the roughness parameters $\left(R_{q}\right.$ and $\lambda_{q}$ ) of the cathode surface, then substitute $a=\sqrt{2} R_{q}$ and $k=2 \pi / \lambda_{q}$ into Eq. (20), the result would be the upper limit of the roughness emittance growth of the real cathode, as shown in Eq. (27):

$$
\bar{\varepsilon}_{n, x}^{2}=\sigma_{x}^{2} \cdot\left[\frac{1}{3 c}\left(\hbar \omega-\phi_{\mathrm{eff}}\right)+\frac{\pi e^{2}}{c} \cdot \frac{R_{q}^{2} E}{\lambda_{q}}\right] .
$$


As shown in Fig. 13, both the 3D and matched 2D surface do not support a significant roughness emittance growth $(\geq 10 \%)$ even when the applied field strength is approaching $150 \mathrm{MV} / \mathrm{m}$. This fact proves that the large emittance growth observed in some experiments might not be mainly caused by the surface roughness and the high rf field, there could be another main factor.

\section{SUMMARY}

In this paper, we introduced the point spread function of photocathode, used it to check the validity of the momentum-position independent assumption, then derived the analytical formulas for emittance growth caused by roughness of a random 2D or 3D surface. Through a simple but effective 2D sinusoidal surface case, we discussed the amount of the cross term of smooth emittance and roughness emittance and pointed out that it is inappropriate to ignore this cross term when calculating the total emittance.

Then we performed numerical simulation on the emittance growth due to roughness of a real-life cathode, and surprisingly found that in the 3D random surface case and the matched 2D sinusoidal surface case, the influence of the surface roughness to the emittance growth is much smaller than expected. Even with the applied electric field strength up to $120 \mathrm{MV} / \mathrm{m}$, the total emittance growth factor is still under 1.1 , which is contradicted to the large emittance growth factor of 1.5-2 observed in some experiments. Therefore there might be some other factors for the growth of the emittance. The study on the unknown factors is ongoing.

\section{ACKNOWLEDGMENTS}

I would like to thank X. L. XU for many productive discussions on math and physics, and thank W. B. LI for his assistance on measuring the topography of photocathodes. I also very much appreciate the helpful suggestions and comments from H. J. Qian, R. K. Li and D. Wang. This work was supported by the National Basic Research Program of China (No. 2011CB808302).

\section{APPENDIX A: DERIVATION OF PSF}

As described in Eq. (5), there are relations between coordinate $(s, \theta, \phi, E, \omega)$ and coordinate $\left(x, y, p_{x}, p_{y}, p_{z}\right)$. Unfortunately, from Eq. (5) one could see that there is no one-to-one correspondence between the two coordinate system, thus the Jacobian determinant of these two coordinates must be zero, which means that the distribution function under the two coordinates could not be connected directly through the Jacobian determinant. Considering the axisymmetric of the electron phase-space distribution, we will use a media coordinate $\left(r, p_{r}, p_{z}\right)$ to play a trick.

Integrating $f(s, \theta, \phi, E, \omega)$ over $\phi$, we get the formation that $f(s, \theta, E ; \omega)=2 \pi C e^{-\frac{s}{\lambda}} \sin \theta$. Then letting $p=\sqrt{2 m(E+\hbar \omega)}$, we could write down the following equations that connect $(s, \theta, E)$ and $\left(r, p_{r}, p_{z}\right)$ :

$$
\begin{aligned}
r & =s \tan \theta, \\
p_{r} & =p \sin \theta, \\
p_{z} & =\sqrt{p^{2}-p_{r}^{2}-p_{m}^{2}} .
\end{aligned}
$$

The Jacobian determinant of these two coordinates is

$$
\begin{aligned}
|J| & =\left|\begin{array}{ccc}
0 & \frac{p_{r}}{m} & \frac{p_{z}}{m} \\
\frac{\sqrt{p_{z}^{2}+p_{m}^{2}}}{p_{r}} & -\frac{r \sqrt{p_{z}^{2}+p_{m}^{2}}}{p_{r}^{2}} & \frac{r}{p_{r}} \frac{p_{z}}{\sqrt{p_{z}^{2}+p_{m}^{2}}} \\
0 & \frac{\sqrt{p_{z}^{2}+p_{m}^{2}}}{p_{r}^{2}+p_{z}^{2}+p_{m}^{2}} & -\frac{p_{r} p_{z}}{\sqrt{p_{z}^{2}+p_{m}^{2}}\left(p_{r}^{2}+p_{z}^{2}+p_{m}^{2}\right)}
\end{array}\right| \\
& =\frac{1}{m} \cdot \frac{p_{z}}{p_{r}} .
\end{aligned}
$$

So that $d E d s d \theta=|J| d r d p_{r} d p_{z}$. Thus we got the PSF under $\left(r, p_{r}, p_{z}\right)$ coordinate system:

$$
\begin{aligned}
f\left(r, p_{r}, p_{z}\right)= & C \exp \left[-\frac{\sqrt{p_{z}^{2}+p_{m}^{2}}}{p_{r}} \cdot \frac{r}{\lambda}\right] \cdot \frac{p_{z}}{\sqrt{p_{r}^{2}+p_{z}^{2}+p_{m}^{2}}} \\
& \cdot H\left(p_{r}\right) H\left(p_{z}\right) H(r) H\left(p_{M}^{2}-p_{m}^{2}-p_{r}^{2}-p_{z}^{2}\right) .
\end{aligned}
$$

Now we will make good use of the axisymmetric of this problem to transfer the PSF from $\left(r, p_{r}, p_{z}\right)$ coordinate to $\left(x, y, p_{x}, p_{y}, p_{z}\right)$ coordinate. Assume that PSF in the $\left(x, y, p_{x}, p_{y}, p_{z}\right)$ coordinate has the general form of

$f\left(x, y, p_{x}, p_{y}, p_{z}\right)=g\left(r, p_{r}, p_{z}\right) \delta\left(x p_{y}-y p_{x}\right) H\left(x p_{x}\right)$.

By using the integration equivalent relation,

$$
\begin{aligned}
& d p_{z} \iint_{p_{r}}^{p_{r}+d p_{r}} d p_{x} d p_{y} \iint_{r}^{r+d r} d x d y f\left(x, y, p_{x}, p_{y}, p_{z}\right) \\
& \quad=f\left(r, p_{r}, p_{z}\right) d r d p_{r} d p_{z} .
\end{aligned}
$$

We get the relation between $g\left(r, p_{r}, p_{z}\right)$ and $f\left(r, p_{r}, p_{z}\right)$,

$$
g\left(r, p_{r}, p_{z}\right)=\frac{1}{2 \pi} f\left(r, p_{r}, p_{z}\right) .
$$

Substitute $r=\sqrt{x^{2}+y^{2}}$ and $p_{r}=\sqrt{p_{x}^{2}+p_{y}^{2}}$ into Eq. (A4), we would finally obtain the PSF as Eq. (6).

\section{APPENDIX B: STATISTIC ON MOMENTUMS OF EMITTED ELECTRONS ON A SMOOTH PHOTOCATHODE}

There are lots of statistics on electron momentums in this paper, so it is necessary to evaluate them for future use. 
Note that we constrain our discussion on smooth surface photocathode which irradiated by normal incident laser beam.

The electron distribution of this case is

$$
\begin{aligned}
& D\left(x, y, p_{x}, p_{y}, p_{z}\right) \\
& \quad=C \cdot \frac{p_{z}}{\sqrt{p_{z}^{2}+p_{m}^{2}} \cdot \sqrt{p_{x}^{2}+p_{y}^{2}+p_{z}^{2}+p_{m}^{2}}} .
\end{aligned}
$$

Define $\Delta=p_{M}-p_{m}$ and $\delta=\left(p_{M}-p_{m}\right) / p_{m}$, using the electron distribution of Eq. (B1), one could get the statistic parameters on the momentum of emitted electrons as below:

$$
\begin{aligned}
& \left\langle p_{x}\right\rangle=0, \\
& \left\langle p_{y}\right\rangle=0, \\
& \left\langle p_{z}\right\rangle \approx p_{m}\left[\frac{8 \sqrt{2}}{15} \sqrt{\delta}+\frac{2 \sqrt{2}}{35}(\sqrt{\delta})^{3}\right], \\
& \left\langle p_{x}^{2}\right\rangle=\frac{1}{6} \Delta\left(\Delta+2 p_{m}\right), \\
& \left\langle p_{y}^{2}\right\rangle=\frac{1}{6} \Delta\left(\Delta+2 p_{m}\right), \\
& \left\langle p_{z}^{2}\right\rangle=\frac{1}{6} \Delta\left(\Delta+4 p_{m}\right) .
\end{aligned}
$$

It should be noted that these formulas could only be used in a 3D, bulk emission metallic smooth photocathode when irradiated by a normal incident laser beam.

\section{APPENDIX C: LOCAL DISTRIBUTION TO GLOBAL DISTRIBUTION}

Substituting Eq. (15) to Eq. (16) one would obtain the form of local distribution:

$$
\begin{aligned}
D^{\prime}= & I\left(x_{0}, y_{0}\right) \cos \theta \\
& \times \frac{C p_{z}^{\prime}}{\sqrt{p_{z}^{\prime 2}+p_{m}^{2}} \cdot \sqrt{p_{x}^{\prime 2}+p_{y}^{\prime 2}+p_{z}^{\prime 2}+p_{m}^{2}}} .
\end{aligned}
$$

Assume that the transform matrix between local and global coordinate is

$$
\mathbf{D}=\left(\begin{array}{c}
\mathbf{m} \\
\mathbf{n} \\
\mathbf{k}
\end{array}\right) .
$$

Then the following relation would be satisfied:

$$
\mathbf{D} \cdot\left(\begin{array}{c}
p_{x} \\
p_{y} \\
p_{z}
\end{array}\right)=\left(\begin{array}{c}
p_{x}^{\prime} \\
p_{y}^{\prime} \\
p_{z}^{\prime}
\end{array}\right) .
$$

Because we did not specify the directions of local coordinate $S^{\prime}$, unit vector $\boldsymbol{m}$ and $\boldsymbol{n}$ could be freely picked (while keeping them perpendicular to each other and to $\boldsymbol{k}$ ). But $\boldsymbol{k}$ should be the normal vector of point $\mathbf{P}$, thus $\boldsymbol{k}$ satisfies

$$
\begin{aligned}
\mathbf{k}= & \left(\frac{-\partial_{x} R}{\sqrt{1+\partial_{x}^{2} R+\partial_{y}^{2} R}}, \frac{-\partial_{y} R}{\sqrt{1+\partial_{x}^{2} R+\partial_{y}^{2} R}},\right. \\
& \left.\frac{1}{\sqrt{1+\partial_{x}^{2} R+\partial_{y}^{2} R}}\right) .
\end{aligned}
$$

Substituting Eq. (C4) into Eq. (C3), we would get $\left(p_{x}^{\prime}, p_{y}^{\prime}, p_{z}^{\prime}\right)$ as a function of $\left(p_{x}, p_{y}, p_{z}\right)$. Replace all the $p^{\prime}$ terms to $p$ in Eq. (C1), with consideration of the axisymmetric of the local coordinate and using results from Appendix B, after a certain amount of calculations one could get the form shown in Eq. (17).

\section{APPENDIX D: MISCELLANEOUSNESS}

\section{Derivation for Eq. (9)}

To get a clear idea where Eq. (9) comes from, let us simplify the $3 \mathrm{D}$ case to the $2 \mathrm{D}$ case, so that expression $v\left(x, y, p_{x}, p_{y}, p_{z}\right)$ becomes $v\left(x, p_{x}\right)$, the intensity distribution function of the laser becomes $I(x)$, and the PSF becomes $f\left(x, p_{x}\right)$.

Now let us do the statistic:

$$
\begin{aligned}
\langle v\rangle_{D} & =\langle v\rangle_{I * f}=\iint d x d p_{x} v\left(x, p_{x}\right) \cdot I * f\left(x, p_{x}\right) \\
& =\iint d x d p_{x} v\left(x, p_{x}\right) \int d x^{\prime} I\left(x^{\prime}\right) \cdot f\left(x-x^{\prime}, p_{x}\right)
\end{aligned}
$$

Let $x_{I}=x^{\prime}, x_{f}=x-x^{\prime}$

$$
\begin{aligned}
& =\iint d x_{f} d p_{x} f\left(x_{f}, p_{x}\right) \int d x_{I} I\left(x_{I}\right) \cdot v\left(x_{I}+x_{f}, p_{x}\right) \\
& =\left\langle\left\langle v\left(x_{I}+x_{f}, p_{x}\right)\right\rangle_{I}\right\rangle_{f}=\left\langle\left\langle v\left(x_{I}+x_{f}, p_{x}\right)\right\rangle_{f}\right\rangle_{I} \\
& =\left\langle v\left(x_{I}+x_{f}, p_{x}\right)\right\rangle_{I \cdot f} .
\end{aligned}
$$

Note that $d x d x^{\prime}=d x_{I} d x_{f}$ since the Jacobian determinant of the transfer from $\left(x, x^{\prime}\right)$ to $\left(x_{f}, x_{I}\right)$ is 1 . The above derivation shows how Eq. (9) comes.

\section{Single point emittance}

We attempt to prove that for single point incidence (all photons inject into the same position on the smooth surface), if $\hbar \omega$ is close to $\phi_{\text {eff }}$, the emittance $\varepsilon_{f}$ would be

$$
\varepsilon_{f} \approx \frac{1}{3} \lambda\left(p_{M}-p_{m}\right)
$$

where $p_{m}, p_{M}$ and $\lambda$ keep the same definition as shown in Eq. (4). 
By applying the statistical emittance formula, we get

$$
\varepsilon_{f}^{2}=\left\langle x^{2}\right\rangle_{f}\left\langle p_{x}^{2}\right\rangle_{f}-\left\langle x p_{x}\right\rangle_{f}^{2} .
$$

To evaluate the formula above, there are at least two ways: (i) applying the PSF Eq. (6) directly; (ii) applying the original probability density function Eq. (3). Here we will go through way (ii) since it seems more friendly to human beings.

For simplification, let $p=\sqrt{2 m(E+\hbar \omega)}$ and $\cos \theta_{M}=$ $p_{m} / p$, also let $\Delta=p_{M}-p_{m}$ and $\delta=\left(p_{M}-p_{m}\right) / p_{m}$, applying Eq. (3), we get

$$
\begin{aligned}
& \left\langle x^{2}\right\rangle_{f}=\frac{\int_{p_{m}}^{p_{M}} p d p \int_{0}^{\infty} d s \int_{0}^{2 \pi} d \phi \int_{0}^{\theta_{M}} d \theta(s \tan \theta \cos \phi)^{2} e^{-\frac{s}{\lambda}} \sin \theta}{\int_{p_{m}}^{p_{M}} p d p \int_{0}^{\infty} d s \int_{0}^{2 \pi} d \phi \int_{0}^{\theta_{M}} d \theta e^{-\frac{s}{\lambda}} \sin \theta} \\
& =\frac{\int_{p_{m}}^{p_{M}} p d p \int_{0}^{\infty} s^{2} e^{-\frac{s}{\lambda}} d s \int_{0}^{2 \pi} \cos ^{2} \phi d \phi \int_{0}^{\theta_{M}} \tan ^{2} \theta \sin \theta d \theta}{\int_{p_{m}}^{p_{M}} p d p \int_{0}^{\infty} e^{-\frac{s}{\lambda}} d s \int_{0}^{2 \pi} d \phi \int_{0}^{\theta_{M}} \sin \theta d \theta} \\
& =\frac{\int_{p_{m}}^{p_{M}} p d p \cdot \lambda^{3} \Gamma(3) \cdot \pi \cdot\left(p / p_{m}+p_{m} / p-2\right)}{\int_{p_{m}}^{p_{M}} p d p \cdot \lambda \Gamma(1) \cdot 2 \pi \cdot\left(1-p_{m} / p\right)} \\
& =\frac{\lambda^{2}}{p_{m}} \cdot \frac{\Delta^{3} / 3}{\Delta^{2} / 2}=\frac{2}{3} \lambda^{2} \delta, \\
& \left\langle p_{x}^{2}\right\rangle_{f}=\frac{\int_{p_{m}}^{p_{M}} p d p \int_{0}^{\infty} d s \int_{0}^{2 \pi} d \phi \int_{0}^{\theta_{M}} d \theta(p \sin \theta \cos \phi)^{2} e^{-\frac{s}{\lambda}} \sin \theta}{\int_{p_{m}}^{p_{M}} p d p \int_{0}^{\infty} d s \int_{0}^{2 \pi} d \phi \int_{0}^{\theta_{M}} d \theta e^{-\frac{s}{\lambda}} \sin \theta} \\
& =\frac{\int_{p_{m}}^{p_{M}} p^{3} d p \int_{0}^{\infty} e^{-\frac{s}{\lambda}} d s \int_{0}^{2 \pi} \cos ^{2} \phi d \phi \int_{0}^{\theta_{M}} \sin ^{3} \theta d \theta}{\int_{p_{m}}^{p_{M}} p d p \int_{0}^{\infty} e^{-\frac{s}{\lambda}} d s \int_{0}^{2 \pi} d \phi \int_{0}^{\theta_{M}} \sin \theta d \theta} \\
& =\frac{\int_{p_{m}}^{p_{M}} p^{3} d p \cdot \lambda \Gamma(1) \cdot \pi \cdot\left(2 / 3-p_{m} / p+1 / 3 \cdot p_{m}^{3} / p^{3}\right)}{\int_{p_{m}}^{p_{M}} p d p \cdot \lambda \Gamma(1) \cdot 2 \pi \cdot\left(1-p_{m} / p\right)} \\
& =\frac{1}{2} \cdot \frac{\Delta^{4} / 6+p_{m} \cdot \Delta^{3} / 3}{\Delta^{2} / 2}=\frac{1}{6} \Delta\left(\Delta+2 p_{m}\right), \\
& \left\langle x p_{x}\right\rangle_{f}=\frac{\int_{p_{m}}^{p_{M}} p d p \int_{0}^{\infty} d s \int_{0}^{2 \pi} d \phi \int_{0}^{\theta_{M}} d \theta\left(s p \tan \theta \sin \theta \cos ^{2} \phi\right) e^{-\frac{s}{\lambda}} \sin \theta}{\int_{p_{m}}^{p_{M}} p d p \int_{0}^{\infty} d s \int_{0}^{2 \pi} d \phi \int_{0}^{\theta_{M}} d \theta e^{-\frac{s}{\lambda}} \sin \theta} \\
& =\frac{\int_{p_{m}}^{p_{M}} p^{2} d p \int_{0}^{\infty} s e^{-\frac{s}{\lambda}} d s \int_{0}^{2 \pi} \cos ^{2} \phi d \phi \int_{0}^{\theta_{M}} \tan \theta \sin ^{2} \theta d \theta}{\int_{p_{m}}^{p_{M}} p d p \int_{0}^{\infty} e^{-\frac{s}{\lambda}} d s \int_{0}^{2 \pi} d \phi \int_{0}^{\theta_{M}} \sin \theta d \theta} \\
& =\frac{\int_{p_{m}}^{p_{M}} p^{2} d p \cdot \lambda^{2} \Gamma(2) \cdot \pi \cdot\left(-1 / 2-\ln \left(p_{m} / p\right)+1 / 2 \cdot p_{m}^{2} / p^{2}\right)}{\int_{p_{m}}^{p_{M}} p d p \cdot \lambda \Gamma(1) \cdot 2 \pi \cdot\left(1-p_{m} / p\right)} \\
& =\frac{\lambda}{2} \cdot \frac{\frac{1}{3} p_{M}^{3} \ln \left(p_{M} / p_{m}\right)-\frac{1}{9}\left(p_{M}^{3}-p_{m}^{3}\right)-\frac{1}{6} \Delta^{2}\left(p_{M}+2 p_{m}\right)}{\Delta^{2} / 2} \\
& =\frac{1}{3} \lambda \Delta\left(1+\frac{1}{4} \delta+O\left(\delta^{2}\right)\right) \approx \frac{1}{3} \lambda \Delta .
\end{aligned}
$$

Substituting all the results above into the statistical emittance formula, we get

$$
\begin{aligned}
\varepsilon_{f} & =\sqrt{\left\langle x^{2}\right\rangle_{f}\left\langle p_{x}^{2}\right\rangle_{f}-\left\langle x p_{x}\right\rangle_{f}^{2}} \approx \frac{1}{3} \lambda \sqrt{\delta \cdot \Delta\left(\Delta+2 p_{m}\right)-\Delta^{2}} \\
& =\frac{1}{3} \lambda \sqrt{\delta \cdot \Delta^{2}+2 \Delta^{2}-\Delta^{2}}=\frac{1}{3} \lambda \sqrt{\Delta^{2} \cdot(1+\delta)} \\
& \approx \frac{1}{3} \lambda \Delta=\frac{1}{3} \lambda\left(p_{M}-p_{m}\right) .
\end{aligned}
$$

That is what we want to prove. 
[1] P. Emma, R. Akre, J. Arthur, R. Bionta, C. Bostedt, J. Bozek, A. Brachmann, P. Bucksbaum, R. Coffee, F.-J. Decker et al., Nat. Photonics 4, 641 (2010).

[2] J. Corlett, A next generation light source facility at lbnl, in Proceedings of the 24th Particle Accelerator Conference, PAC-2011, New York, 2011 (IEEE, New York, 2011).

[3] B. Patterson, R. Abela, H. Braun, U. Flechsig, R. Ganter, Y. Kim, E. Kirk, A. Oppelt, M. Pedrozzi, S. Reiche et al., New J. Phys. 12, 035012 (2010).

[4] R. Schoenlein, W. Leemans, A. Chin, P. Volfbeyn, T. Glover, P. Balling, M. Zolotorev, K. Kim, S. Chattopadhyay, and C. Shank, Science 274, 236 (1996).

[5] P. Musumeci, J. Moody, and C. Scoby, Ultramicroscopy 108, 1450 (2008).

[6] D. H. Dowell and J. F. Schmerge, Phys. Rev. ST Accel. Beams 12, 074201 (2009).

[7] D. Dowell, F. King, R. Kirby, J. Schmerge, and J. Smedley, Phys. Rev. ST Accel. Beams 9, 063502 (2006).

[8] O. Renault, R. Brochier, A. Roule, P.-H. Haumesser, B. Krömker, and D. Funnemann, Surf. Interface Anal. 38, 375 (2006).

[9] H. Qian, Research on the emittance issues of photocathode rf gun, Ph.D. thesis, Tsinghua University, 2012.

[10] H. Qian, Phys. Rev. ST Accel. Beams 15, 040102 (2012).

[11] F. Le Pimpec, C. Milne, C. Hauri, and F. Ardana-Lamas, Appl. Phys. A 112, 647 (2013).
[12] X. He, C. Tang, W. Huang, and Y. Lin, High Energy Phys. Nucl. Phys. 28, 1007 (2004).

[13] K. Flottmann (1997).

[14] Y. Y. Lau, J. Appl. Phys. 61, 36 (1987).

[15] M. Krasilnikov, Impact of the cathode roughness on the emittance of an electron beam, in Proceedings of FEL 2006 Conference, Berlin, Germany (BESSY, Berlin, 2006), pp. 583-586.

[16] X. He, Theoretical and initial experimental research on low emittance photoinjector, Ph.D. thesis, Tsinghua University, 2006.

[17] D. Xiang, W.-H. Huang, Y.-C. Du, L.-X. Yan, R.-K. Li, C.-X. Tang, Y.-Z. Lin, S.-J. Park, and J. Park, First principle measurements of thermal emittance for copper and magnesium, in Proceedings of the 22nd Particle Accelerator Conference, PAC-2007, Albuquerque, NM (IEEE, New York, 2007), pp. 1049-1051.

[18] L. Serafini and J. B. Rosenzweig, Phys. Rev. E 55, 7565 (1997).

[19] To be more accurate, help us understand why the method we use to calculate the roughness emittance is correct.

[20] R. W. Klopfenstein and R. K. Wehner, RCA Rev. 34, 630 (1973).

[21] E. Gadelmawla, M. Koura, T. Maksoud, I. Elewa, and H. Soliman, J. Mater. Process. Technol. 123, 133 (2002). 